\title{
Layer Morphology Analysis of Sputter-eroded Silicon Gratings using Rutherford Backscattering
}

\author{
Hagen Langhuth, Matej Mayer*, Stefan Lindig \\ Max-Planck-Institut für Plasmaphysik, EURATOM Association, Boltzmannstr. 2, \\ 85748 Garching, Germany
}

\begin{abstract}
The possibility to simulate Rutherford backscattering (RBS) spectra from arbitrary laterally inhomogeneous sample structures was implemented in the SIMNRA code. Layer morphology is modeled by a layer thickness frequency distribution. This method was used to monitor the evolution of the surface morphology of a one dimensional silicon grating on top of a tantalum interlayer in situ. The Si grating was sputtered by argon and carbon ion beams at an incident energy of $6 \mathrm{keV}$ at two different angles of incidence parallel to the grid lines. After each sputtering step the surface was investigated by RBS. The Si grid lines undergo a change of their morphology due to sputtering erosion. The morphology change depends on the sputtering angle and is different for Ar and C bombardment. Sputtering with $\mathrm{C}$ leads to the formation of a protective $C$ layer on top, which was confirmed by additional NRA measurements. The results of the RBS measurements were confirmed by scanning electron micrographs of sample cross-sections produced by focused ion beam cross-sectioning.
\end{abstract}

Keywords

Surface morphology, in situ RBS, SIMNRA 
* Corresponding author: Matej Mayer, Max-Planck-Institut für Plasmaphysik, Boltzmannstr. 2, 85748 Garching, Germany, Tel.: +49/89/3299-1639, Fax: +49/89/3299-2279, Email: Matej.Mayer@ipp.mpg.de 


\section{Introduction}

Ion Beam Analysis (IBA) methods such as Rutherford backscattering (RBS) are usually considered as methods for measuring the depth profiles of different elements $[1,2]$. It is often overlooked, however, that these methods measure a depth profile only for laterally homogeneous samples. In the case of laterally inhomogeneous samples with homogeneous, but laterally varying layer thickness, these methods do not determine depth profiles of elements, but provide information about the layer thickness distribution, i.e. the layer morphology. A random thickness distribution is usually called layer roughness, and it has been already demonstrated that roughness parameters (such as the layer thickness variation) can be measured with IBA methods [3]. The possibility to derive the layer thickness distribution in general cases was already shown in $[4,5,6,7,8,9,10,11]$.

Layer thickness distributions are usually investigated by imaging methods, such as atomic force microscopy (AFM) of the sample surface or scanning electron microscopy (SEM) investigation of the sample cross-section. With AFM only the sample surface is investigated, so that usually only the corrugation of the surface layer, but not the thickness of a layer, can be measured. Properties of buried layers or the composition of a layer cannot be investigated. Moreover, fine and sharp surface structures cannot be resolved due to the finite size of the AFM tip [11]. Cleaving of the sample or producing a cross-section of the surface layer using a focused ion beam (FIB) and investigating the cross-section with a SEM is destructive and provides only limited information about layer composition. These methods are usually used ex-situ and require transfer of the samples through atmosphere.

In this paper we will show that RBS can be used for measuring the morphology of periodic surfaces and the change of this morphology due to sputtering in situ. This method is non-destructive, quantitative for layer thickness and composition, fast, and traceable.

\section{Experimental}

All IBA measurements were performed at the Tandem accelerator in the Dual Beam Experiment (DBE) [12] at the IPP Garching. The sample had a step like patterned 
surface as depicted in figure 1, which shows a cross-section through the layered sample structure. It consists of a silicon substrate, a tantalum intermediate layer with a thickness of $300 \mathrm{~nm}$ and a deposited silicon top layer which shows the grating structure. The He analysis beam had normal incidence. The RBS detector was a solid-state detector with an energy resolution of $15 \mathrm{keV}$ and a solid angle of about $1.58 \mathrm{msr}$ at a scattering angle $\theta=165^{\circ}$. The sample was oriented in such a way that the grating structure was parallel to the exit beam. At this geometry correlation effects, such as incidence through a valley and exit through a hilltop, do not play a role.

The experimental procedure was as follows: After a first IBA analysis of the virgin sample the surface was sputtered by bombardment with Ar or $C$ ions at an energy of $6 \mathrm{keV}$ at an angle $\gamma$. After this sputtering step the sample was analyzed again by the $\mathrm{MeV}$ He beam followed by another sputtering step. This procedure was repeated 7 to 8 times until the top silicon layer completely disappeared (in the case of $\mathrm{Ar}$ sputtering), or until the Si layer thickness did not decrease further (in case of the $\mathrm{C}$ sputtering). Samples sputtered by Ar were analyzed by RBS using a 4 He beam at 2 $\mathrm{MeV}$. Samples sputtered by $\mathrm{C}$ were analyzed by a $3 \mathrm{He}$ beam at $2.5 \mathrm{MeV}$. This enabled us to collect information on the amount of carbon deposited on top of the sample by simultaneous Nuclear Reaction Analysis (NRA) measurements using the $12 \mathrm{C}\left(3 \mathrm{He}, \mathrm{p}_{\mathrm{i}}\right) 14 \mathrm{~N}$ reaction. For the NRA measurement a large-angle proton counter located at a scattering angle of $150^{\circ}$ with a solid angle of $35.94 \mathrm{msr}$ was used. The detector was covered by a $5 \mu \mathrm{m}$ thick $\mathrm{Ni}$ foil, so that only high-energy protons from nuclear reactions were detected. In the DBE installation sputtering and subsequent IBA can be performed in the same setup without breaking the vacuum.

The morphology of the grating steps was investigated additionally by the HELIOS device of the IPP. This is an FEG SEM with focused ion beam (FIB), type Nanolab $600 / F E l$. Here, the focused ion beam was used to produce cross-sections of the surface layer followed by tilting the sample and investigation of the cross section by means of SEM.

Computer simulations were performed using SIMNRA 6.50 [13]. Spectra of the grating structure are calculated by using a linear superposition of sub-spectra, as described for example in [3]. SIMNRA 6.50 allows to use arbitrary layer thickness distribution functions supplied by input file. Correlation effects, such as incidence through a valley and exit through a hilltop or multiple surface crossings, are 
neglected. This is a reasonable approximation for the sample orientation used in the experiments, see above. The code RBS-MAST [7] would allow to handle RBS spectra obtained from 3D-structured samples by taking correlation effects and multiple surface crossings into account, but it does not take the various energyspread contributions (except detector resolution) into account [ 14 ]. This disadvantage, together with the easier usability of SIMNRA and the absence of correlation effects in our experiments, was the main reason to use the simpler approach of a linear superposition of sub-spectra.

\section{Results and discussion}

Figure 2a shows the RBS spectrum of the virgin grating sample (black line) before the first sputtering step. The shape of the curve is dominated by particles backscattered from the tantalum interlayer, which form the broad peak between channels 200 and 1050. The tantalum high-energy edge is in channels 900-1050, the step from channels 950-1000 is due to the Si-grating on top of the Ta. Particles backscattered from the top Si grating are visible in channels 500-700 and overlap with the Ta peak: The information about the top Si grating is therefore mainly contained in the modification of the Ta high energy edge. The steps and peaks marked by the arrows are modifications of the Ta peak by the Si grating on top. The green arrow indicates the influence of the thin parts of the Si grating on the edge at the right side and the peak. The blue arrow shows the influence of the thick parts on the edge and the peak. The red line is a SIMNRA simulation assuming flat and homogeneous layers for the Si substrate, the Ta intermediate layer and the Si top layer. Thus the top Si layer is modeled by its mean thickness only. The simulation does not fit the experimental curve very well since it does not reflect the morphology of the surface at all.

In figure $2 b$ the same spectrum but with a different simulation is shown which fits the recorded spectrum very accurately. This time a distribution of layer thicknesses for the Si top layer has been used. Figure 3 illustrates the procedure to find the correct thicknesses and their statistical weights. In Figure 3a left the cross section of the investigated sample is shown. To model the morphology of the Si top layer we only consider the top layer as seen on the right. All relevant physical processes of RBS 
can be described in the highlighted unit cell (UC) of the grating. The profile of the UC is shown in figure $3 \mathrm{~b}$ left. It can be composed of two distinct layers with thicknesses $h_{i}$ and $h_{j}$. The weighting stems now from the fraction of the area of the respective layer to the total area of the UC. So $h_{i}$ would be weighted with i/UC and $h_{j}$ with $j / U C$. The heights and their statistical weights of a certain UC profile find themselves represented in a frequency distribution as shown to the right of figure $3 \mathrm{~b}$. This way all possible monotone structures can be modeled. In figure $3 \mathrm{c}$ left the right part of the unit cell is still the height $h_{i}$ with the weight of $i / U C$. The triangle can be described as a composition of numerous layers of certain step size. Each one is weighted with the same factor, namely $(j / U C) / x$, where $x$ is the number of steps. The width of the steps is limited by the depth resolution. The weighting is the same for each height since its contribution to the total area is the same. The weighting is true for any triangle which can be spanned between $h_{i}$ and $h_{j}$ with a base of area j. One alternative example is given by the dotted line. The according frequency distribution to all these triangles can be found in figure $3 \mathrm{c}$ right. Hence this method suffers from the surjectivity of the problem: One thickness frequency distribution can stand for several layer thickness profiles. Consequently, for a known thickness frequency distribution the exact thickness profile cannot be determined without additional knowledge. Because RBS measures the frequency distribution, it does not allow the derivation of the layer thickness profile, i.e. an image of the layer in general. However it should be kept in mind that additional knowledge (such as the prior knowledge of symmetry of the sample structure and of the lateral dimensions) may allow to derive unambiguously the thickness profile. To separate between the fundamental geometrical shapes of the profiles (homogeneous thickness, triangular structure, spherical structure) is easily possible, though.

In general the statistical weights for all kinds of profiles can be calculated via the frequency distribution $p(h)$, where $h$ is the layer thickness. It is given by $p(h)=$ $q(x)^{\star} d x / d h$. Here $q(x)$ is the probability for an incident ion to hit a lateral position $x$. For homogeneous incident beam distributions $q(x)=q$ is constant. $d x / d$ is the derivative of the function $x(h)$, i.e. the inverse function of the lateral layer thickness distribution $h(x)$. The integral of $p(h)$ is normalized to unity, in analogy to the area of the unit cell. For the layer thickness profile shown in figure $3 b$ the frequency distribution would be two $\delta$-functions as shown on the right. The resulting frequency distributions are introduced to the program SIMNRA by a file which has to be created by the user. The 
program calculates then the sub-spectra to the respective sub-layers. After that the complete spectrum is composed out of the sub-spectra by linear superposition. This leads to the red curves as shown in figure $2 \mathrm{~b}$.

The DBE experiment allows to perform in situ experiments where the sample is exposed to sputtering and subsequent IBA analysis. This allows to monitor the evolution of the surface morphologies during the sputtering steps. In figure 4 four examples of spectra after sputtering with $\mathrm{Ar}$ ions at $6 \mathrm{keV}$ to different fluences are shown. The spectrum in figure $4 \mathrm{a}$ is taken before the first sputtering step. In figure $4 \mathrm{~b}$ the spectrum after the 1st sputtering step, i.e. after an Ar fluence of 7.37E20 $\mathrm{Ar} / \mathrm{cm}^{\wedge} 2$, is shown. Figure $4 \mathrm{c}$ shows the spectrum after the $3^{\text {rd }}$ sputtering step at a fluence of $2.17 \mathrm{E} 21 \mathrm{Ar} / \mathrm{cm}^{\wedge} 2$. Finally, after the $8^{\text {th }}$ sputtering step with a fluence of $7.74 \mathrm{E} 21 \mathrm{Ar} / \mathrm{cm}^{\wedge} 2$, we find the spectrum shown in figure $4 \mathrm{~d}$. With progressing erosion by sputtering the surface morphology changes. This is indicated by the change of the high-energy tantalum edge (i.e. the step in the spectrum) marked by an arrow in figure $4 a$. After a certain fluence this step has completely disappeared. Also the peaks on top of the Ta peak have disappeared, which means that the silicon top layer has been completely removed by sputtering. The morphology information can be extracted from the frequency distributions which in turn are necessary to fit the spectra with the additional knowledge that the profiles are symmetric (the sputtering is performed at normal incidence) and the lateral extension from SEM. The fitting of the first three spectra shown in figure 4 delivers the first three frequency distributions shown in figure $5 \mathrm{a}$. According to the fit the steps of the grating of the initial specimen are not fully upright, but have a small inclination. This is visible in the frequency distribution, which is not exactly zero between the two $\delta$-functions, and is indicated by the small inclination of the steps for Sputter 0 in Fig. 5b. This inclination results in a slightly steeper increase of the spectrum in channels $950-1000$ and is also visible in FIB cross-sections, see below.

Because RBS measures thicknesses in atoms $/ \mathrm{cm}^{2}$ and SEM in $\mathrm{nm}$, the combination of both measurements gives the true atomic density of the Si grating, which turns out to be $4.28 \mathrm{E} 22 \mathrm{at} / \mathrm{cm}^{\wedge} 3$. This value was used for the conversion of the RBS results to thickness. It is about $14 \%$ smaller than the theoretical Si density: Smaller densities than the theoretical one are not unusual for deposited Si layers [15]. For the case of sputter step 8 no Si was left on the Ta layer. This trivial case has been substituted in 
figure 5 by the situation after sputter step 5 instead of 8 . Transforming the frequency distributions into layer thickness information, one obtains the profiles in figure $5 \mathrm{~b}$. Accepting the assumption of symmetrical sputtering of the Si grid lines, RBSmorphology analysis reveals that the initial rectangular steps transform into trapezoidal domes on top of the thin Si grating. With increasing Ar fluence the trapezoids turn into triangles which shrink as the fluence is increased. Close to the end a more or less flat Si layer remains before disappearing completely, uncovering the Ta intermediate layer at a fluence of $7.74 \mathrm{E} 21 \mathrm{Ar} / \mathrm{cm}^{\wedge} 2$.

It is reasonable to expect the profiles to be symmetrical since the Ar sputter beam hits the surface at normal incidence. But even if the profiles are not symmetrical, the integrals of the frequency distributions would still be correct, which is a valuable information: The correct fit of the curves allows to determine accurately the amount of Si removed by sputtering for the respective fluence. With additional information, like the correct height of the Si top layer in the start, it is also possible to determine the true density of the Si in the top layer.

These results have been confirmed by SEM measurements at the HELIOS device. Figure 6 shows cross-sections of the surface for various sputtering fluences. The images were recorded after the complete erosion of the Si top layer, when no remaining Si was left in the centre of the sputter beam spot. The sputtering beam has a flux profile with a plateau in the beam center and a gradient of decreasing intensity at its edge. Therefore, when moving from the beam center towards outside of the beam spot all stadia of profile shapes between fully removed and completely intact can be found. The cross-sections were obtained by a focused ion beam at different positions between the beam spot center and its outer edge. First the surface was covered by secondary electron-stimulated chemical vapor deposition of a platinum hydrocarbon compound. This layer on top reduces surface effects of the ion beam when finally cutting through the specimen surface leading to sharper cuts and thus to clean profiles of the investigated layers beneath. The compound can be seen in figure 6. It shows examples which match roughly the profiles shown in figure 5, measured by RBS. A perfect match can not be expected since the fluences for figures 5 and 6 are not absolutely identical.

A comparison of figures 5 and 6 can only be made if the sputtering is independent from the flux within the flux variations at the boundary. This was the case for the 
parameters used here. It can be noted that the SEM images from the sputter beam centre confirm that no Si was left on top of the Ta layer (not shown).

In another experiment, the Si grating was sputtered by a carbon beam at an angle of incidence of $\gamma=42^{\circ}$ at $6 \mathrm{keV}$ particle energy. The results of the morphology analysis at different sputtering fluences are shown in figure 7 . The respective spectra were fitted as accurately as in the Ar case at $\gamma=0^{\circ}$ shown in figure 4. At an angle of incidence of at least $42^{\circ}$ parallel to the Si grid the profiles don't seem to evolve via a trapezoidal-socle-shape (like in figure 5 after sputter step 1 or in figure 6b), but their shape remains trapezoidal. This stays true for at least all the shape evolution steps observed in this measurement, including sputter steps 1, 3, ... (not shown). As the sputtering process progresses the hills of the Si layer shrink and finally disappear. A relatively flat Si layer of $170 \mathrm{~nm}$ thickness is left over even for excessively high sputter fluences. The reason for this is the formation of a protective carbon layer at the very surface preventing the Si beneath from further erosion. Since the Si hills disappear it seems that this layer is not formed with sufficient thickness on top of them. The growth of the carbon layer was observed by means of NRA. NRA gives information about the total amount of carbon, but does not give any information about its lateral distribution. We have assumed a homogeneous lateral distribution of carbon for further analysis based on the fact that the carbon incidence was parallel to the silicon grid lines (see Fig. 1). Using the areal density of carbon the fit to the RBS spectra revealed the areal density and thus the morphology of the Si layer. The evolution of the areal density of the Si top layer and of the carbon layer on top of the whole sample is shown in figure 8 as function of the carbon fluence. After a fast decrease of the Si areal density at low carbon fluences the removal rate of the silicon levels off and the silicon areal density does not decrease further at fluences of 9E21 $\mathrm{C} / \mathrm{cm}^{\wedge} 2$ onwards. The areal density of $\mathrm{C}$ is growing steadily, indicating the protective carbon layer formation.

SEM images of the sample after carbon bombardment are shown in figure 9 for comparison. They confirm the observations already made. In figure $9 \mathrm{~b}$ the formation of a carbon layer on top of the Si layer is observed, but only in the valleys between the remaining hilltops. On top of the hills no such layer can be observed, which might be due to their exposure towards the incident beam. For higher fluences the socles shrink and disappear. Finally the Si layer is left over covered by a carbon layer. The 
shapes of the profiles are in very good agreement with the results of the RBS analysis. Although the assumption of a homogeneous carbon coverage, as used for the evaluation of the RBS spectra, is not fully justified from the SEM images, it does not introduce additional severe errors for the profile shape evaluation.

\section{Conclusions}

The possibility to model RBS spectra from laterally inhomogeneous sample structures was implemented in the SIMNRA code. This was used to monitor in situ the erosion and morphology change of silicon gratings on top of a tantalum interlayer bombarded by argon and carbon ions. The method enables us to separate different geometrical shapes of patterned surfaces. Correlations between incident and exit beam are neglected, which requires a proper alignment of the sample, for example by normal incidence and exit of the scattered beam parallel to the grid lines of the grating. The surface profiles derived from the RBS spectra are in very good agreement with SEM investigations of sample cross-sections obtained by focused ion beam cross-sectioning. In the case of argon sputtering the top silicon layer is totally removed at high fluences, while in the case of carbon bombardment the initial hills of the grating are fully removed, while the silicon in the initial valleys remains due to the formation of a protective carbon coating. The technique can be optimized by turning the cumbersome "fitting procedure by hand" into a fully automated process using Bayesian inference.

\section{Acknowledgements}

The authors would like to thank Joachim Dorner and Michael Fußeder for their technical assistance in the ion beam analysis measurements. 


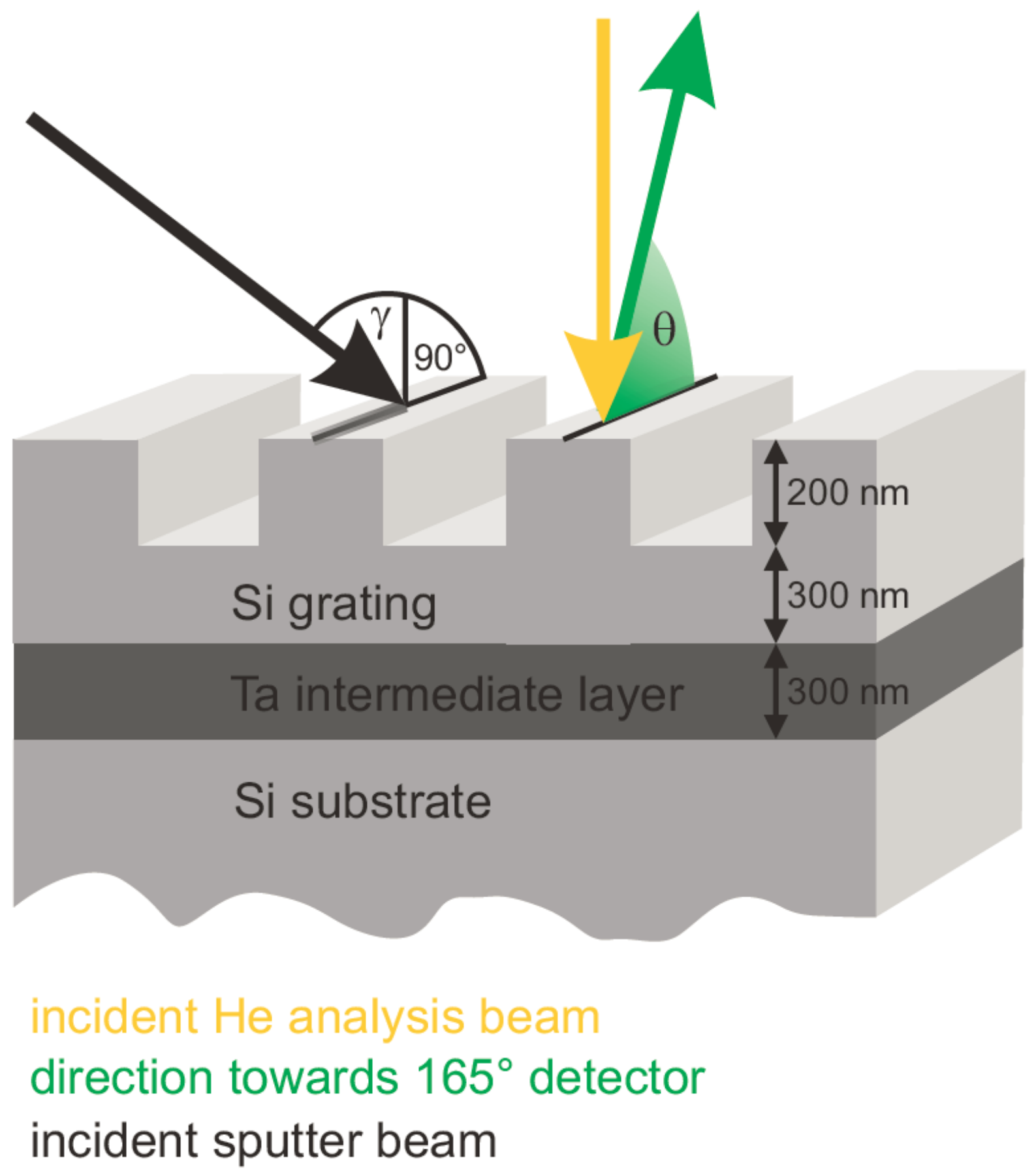

\section{Figure 1:}

Schematic representation of the investigated specimen and the sputtering and IBA geometries. 
a)

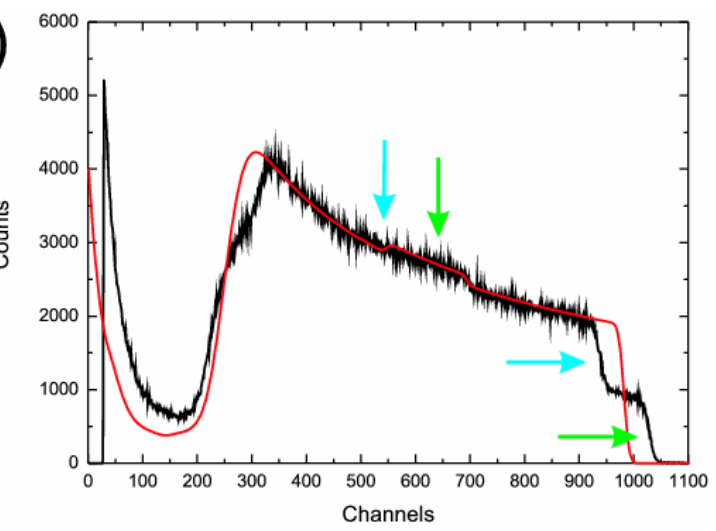

b)

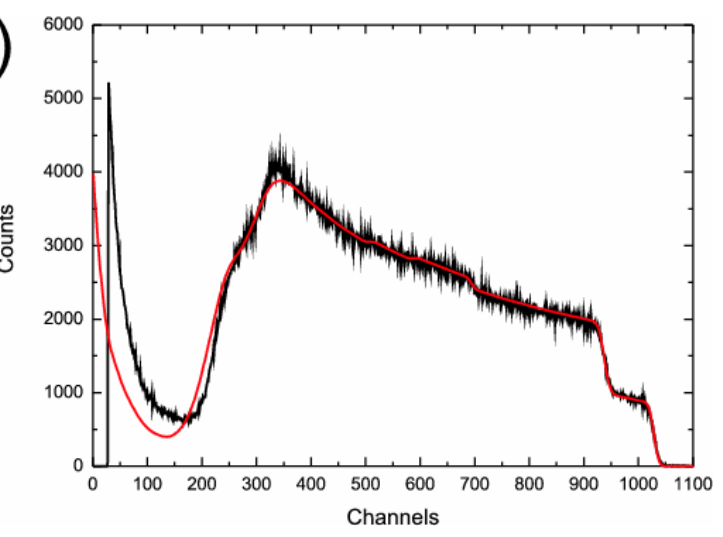

Figure 2:

RBS spectrum of the initial unsputtered specimen (black) with simulated curves (red) under assumption of a mean thickness (a) and of a step like thickness distribution (b). The arrows in a) mark the points where the Si top layer influences the Ta peak dominating the spectrum. 


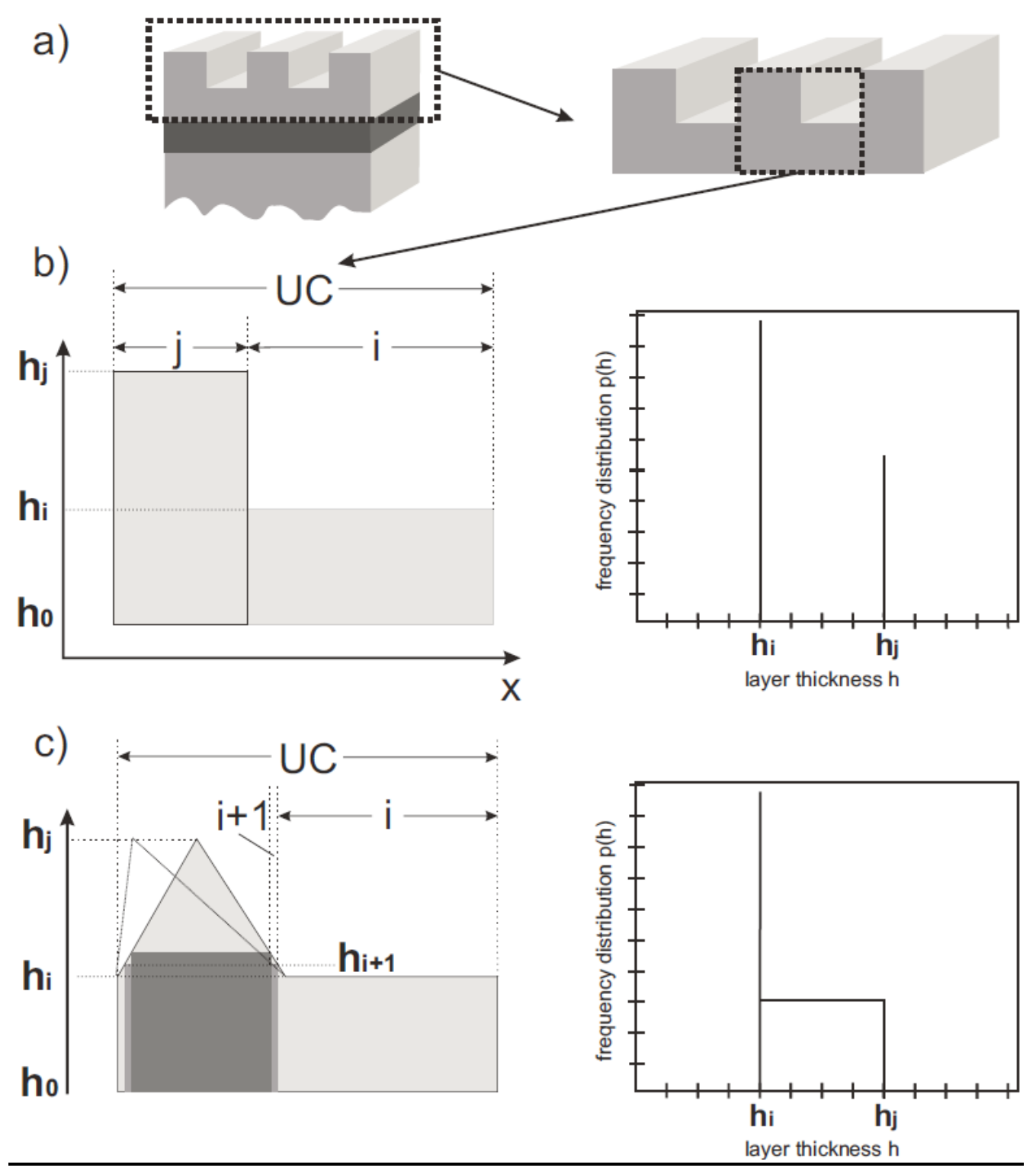

\section{Figure 3:}

Illustration of the procedure how to find the frequency distribution for a given thickness distribution and vice versa. a): Reduction of the periodic structure to the unic cell (UC). b): All monotone thickness profiles, such as the one shown here, can be described by a distribution of layers with associated heights $h_{i}$. i defines the weighting factor of this layer. The structure shown on the left side is shown as frequency distribution of layer thicknesses on the right side. The $\delta$-functions have been replaced by thin box sections, so that the height of each line represents its frequency. c): Another profile shape with its frequency distribution is shown. The triangular shape requires more layers of certain step size to be modeled correctly. The necessary step size is limited by the depth resolution of the RBS. The two triangles on the left produce both the same frequency distribution. 

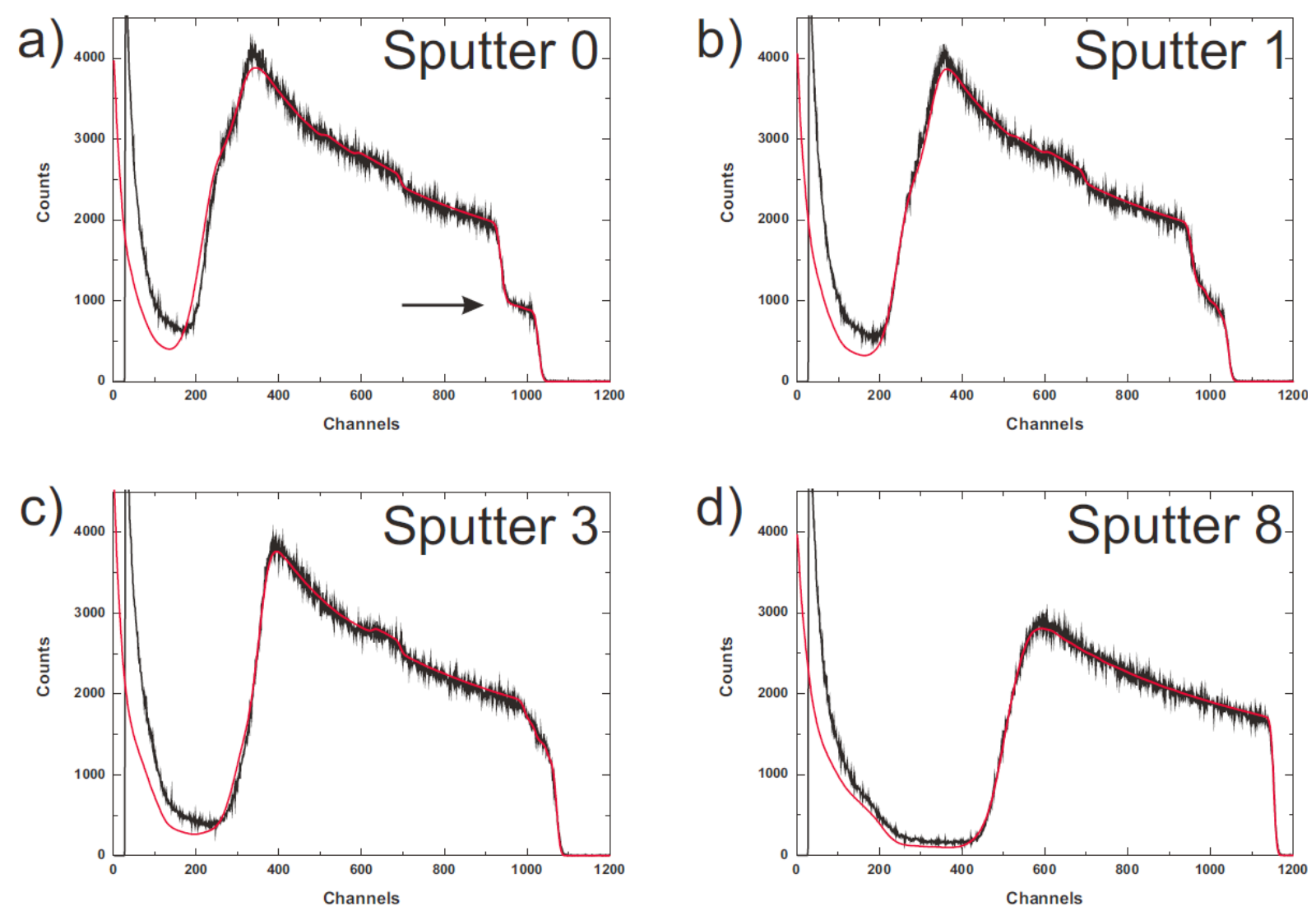

Figure 4:

RBS spectra after bombardment of the Si grating with 4 different sputtering fluences, $6 \mathrm{keV}$ argon ions at normal incidence.. a) Virgin sample (no sputtering). b) after the first sputter step (fluence of 7.37E20 Ar/cm^2), c) after the third (fluence of 2.17E21 $\mathrm{Ar} / \mathrm{cm}^{\wedge} 2$ ) and d) after the eighth sputter step (fluence of 7.74E21 Ar/cm^2). The characteristic step in the spectrum, marked by an arrow in a) disappears with increasing fluence since the Si grid pattern, causing this step in the spectrum, is being removed. 
a)

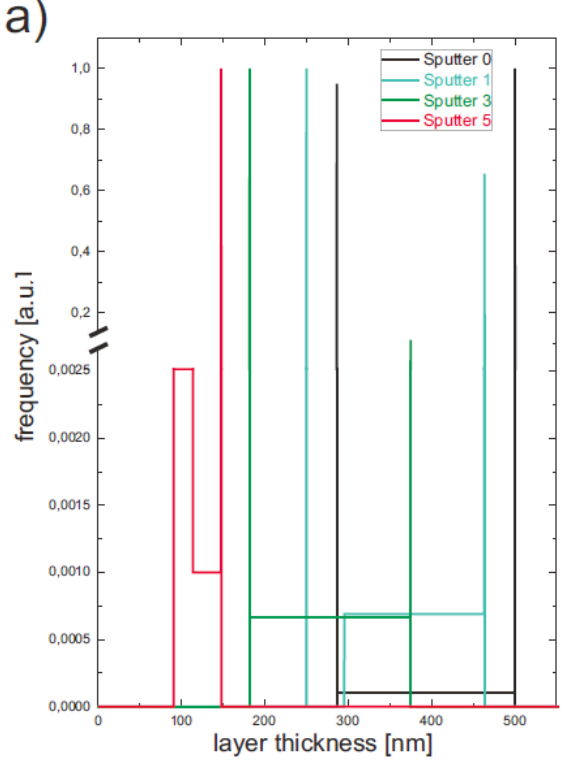

b)

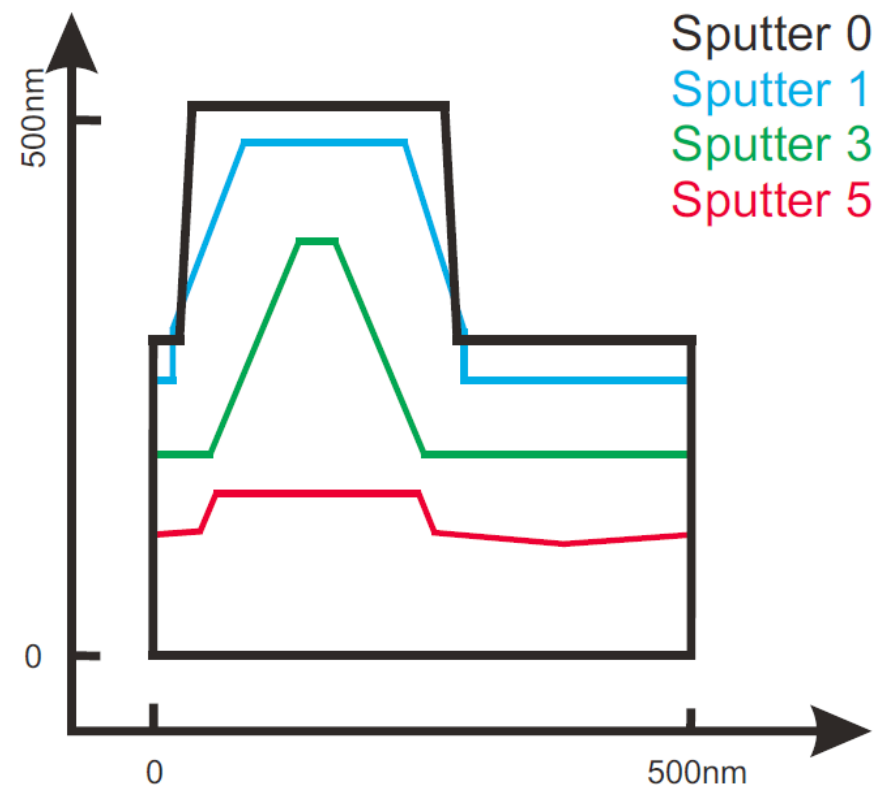

Figure 5:

a): Height frequency distributions, normalized to unity. Derived from the fitting procedure of RBS spectra of the Ar-sputtering steps $0,1,3$ and 5 . The inset at the bottom right highlights that the frequency distribution of sputter step 0 , appearing to be 0 between the two $\delta$-functions, is in fact not 0 . b): Profiles of the Si grid lines for the different Ar-sputtering steps derived from the frequency spectra in a) under the assumption of symmetrical sputtering. 

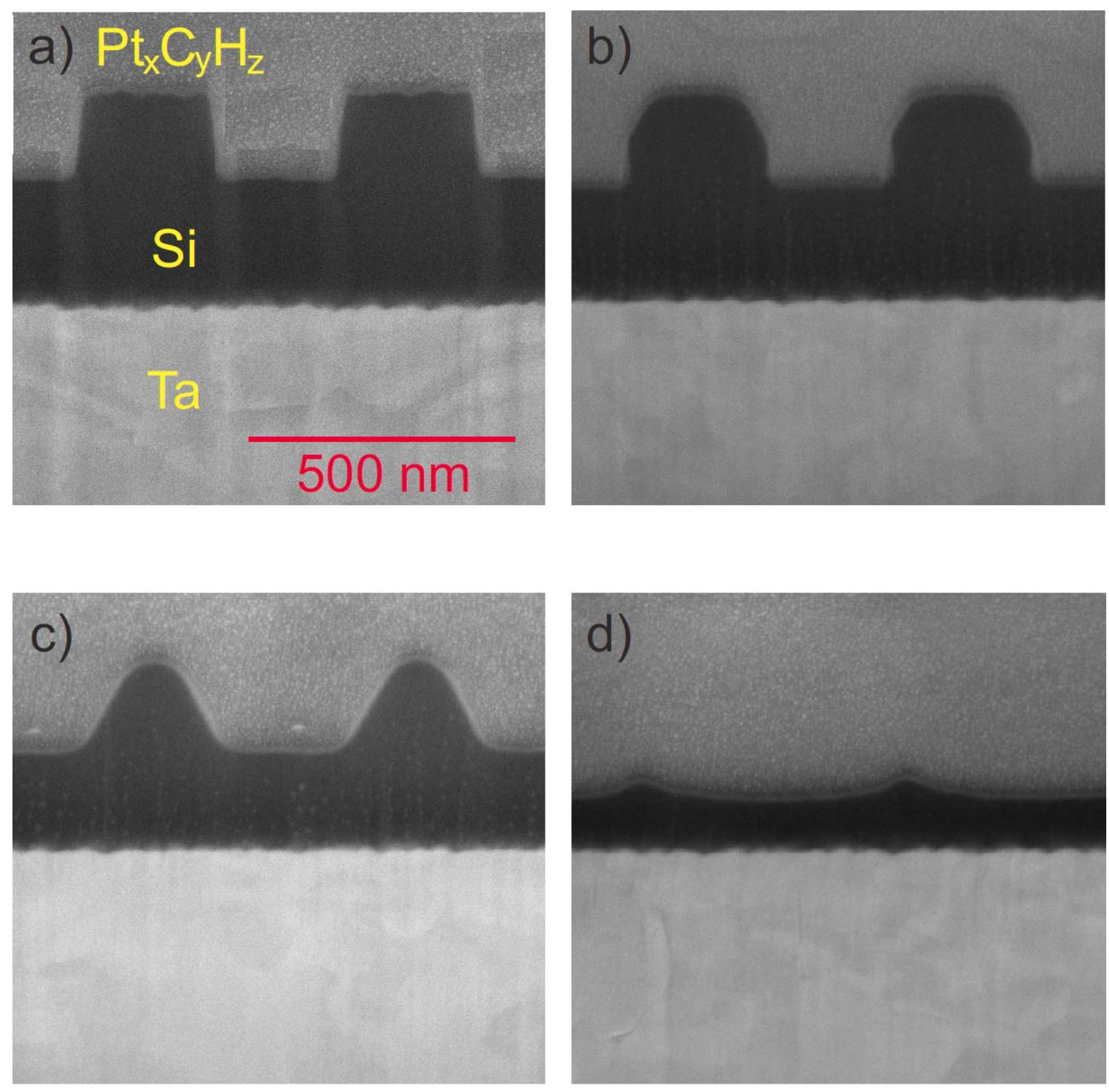

\section{Figure 6:}

Scanning electron micrographs of sample cross-sections produced by FIB. A platinum containing hydrocarbon compound was deposited on the sample surface before FIB cutting. a) Virgin sample, showing the initial profile of the Si grating on top of the Ta layer. b) to d) Sample cross-sections at increasing Ar fluence. 


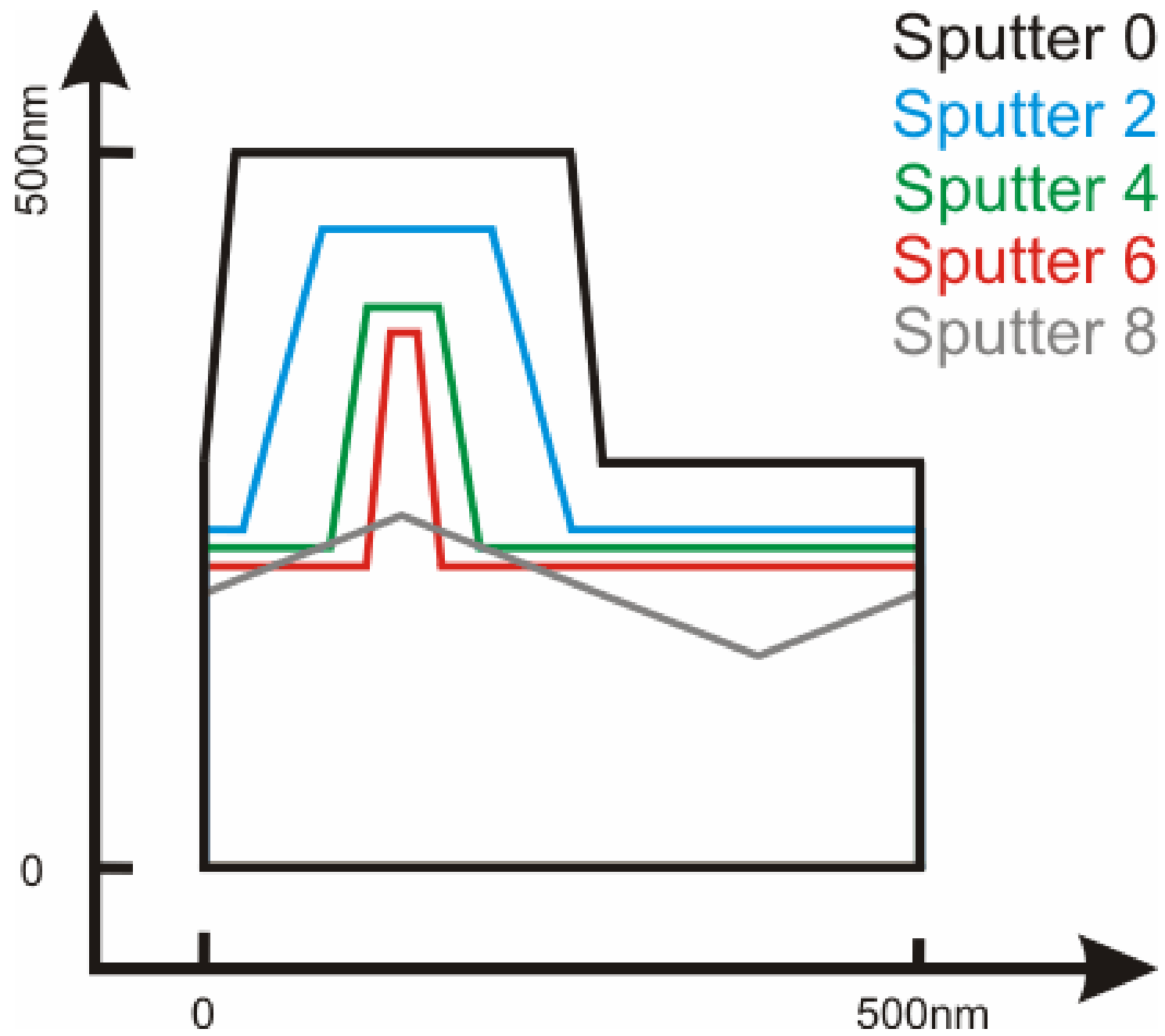

Figure 7:

Thickness profiles of the Si grating for the different C-sputtering steps $0,2,4,6$ and 8. The thickness profiles were derived from the measured frequency distributions analogue to the Ar-case. 

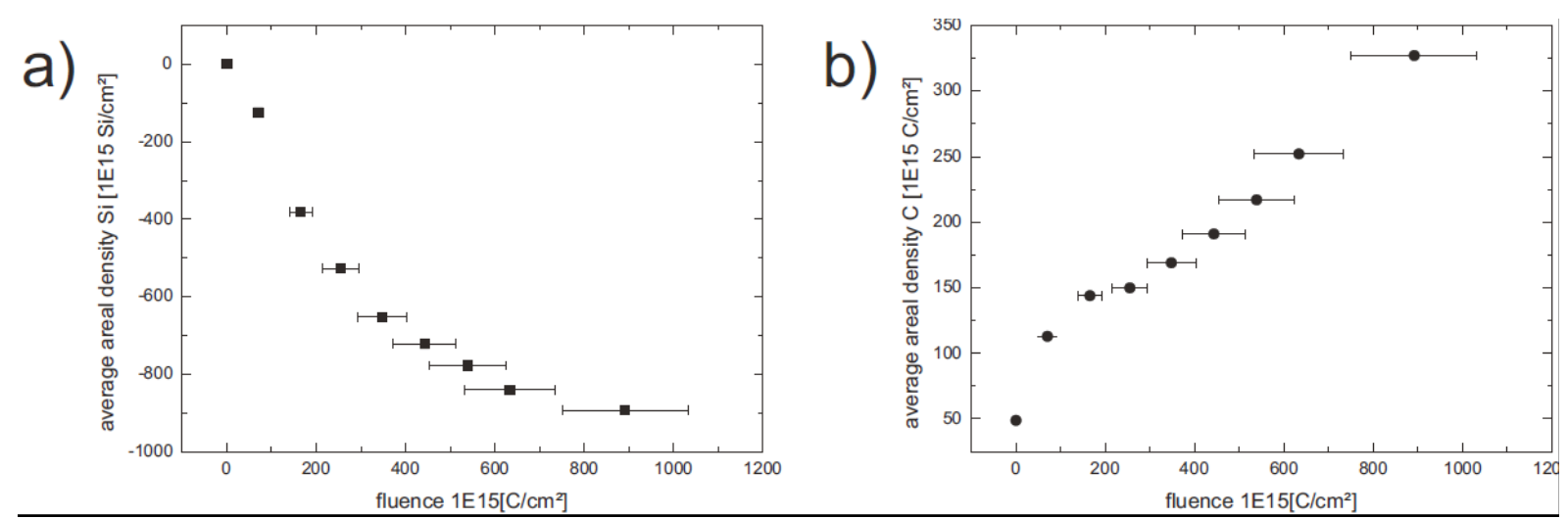

Figure 8:

Average areal densities of the Si top layer (a) and of the deposited C layer (b) as a function of the incident carbon fluence. The areal density of $\mathrm{Si}$ was evaluated by RBS, the amount of $C$ was derived from NRA. 

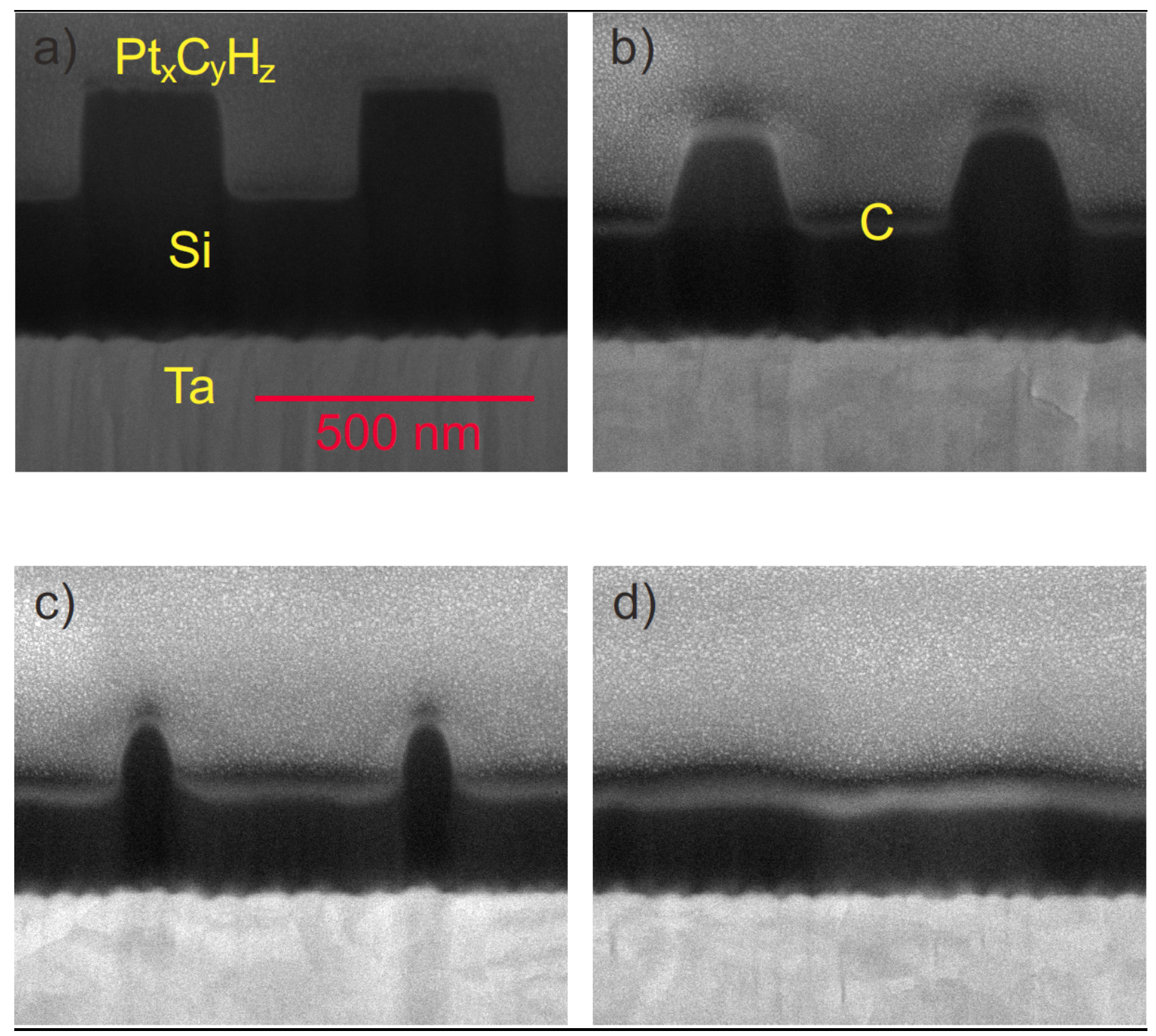

\section{Figure 9:}

SEM images of the C-sputtered specimen. a) Virgin sample, showing the initial profile of the Si grating on top of the Ta layer. b) to d) Sample cross-sections at increasing carbon fluence. In $b$ ) the formation of the protective carbon layer in the valleys can already be identified. As the fluence is increasing, the grid lines disappear and a relatively thick and flat Si layer is left, covered by carbon (d). 
[1] J.R. Tesmer and M. Nastasi, Handbook of Modern Ion Beam Materials Analysis, Materials Research Society, Pittsburgh, Pennsylvania, 1995

[2] Y. Wang and M. Nastasi, Handbook of Modern Ion Beam Materials Analysis, Materials Research Society, Warrendale, Pennsylvania, 2009

[3] M. Mayer, Nucl. Instr. Meth. B194 (2002) 177

[4] X.S. Guo, W.A. Lanford, and K.P. Rodbell, Nucl. Instr. Meth. B45 (1990) 157

[5] P.R. Berning and A. Niiler, Nucl. Instr. Meth. B73 (1993) 178

[6] N. Marin, Y. Serruys, and P. Calmon, Nucl. Instr. Meth. B108 (1996) 179

[7] Z. Hajnal, E. Szilágyi, F. Pászti, and G. Battistig, Nucl. Instr. Meth. B118 (1996) 617

[8] J. Slotte, A. Laakso, T. Ahlgren, E. Rauhala, R. Salonen, J. Räisänen, A. Simon, I. Uzonyi, A.Z. Kiss and E. Somorjai, J. Appl. Phys. 87 (2000) 140

[9] D. Mangelinck, P.S. Lee, T. Osipowitcz, and K.L. Pey, Nucl. Instr. Meth. B 215 (2004) 495

[10] M.J.F. Healy, M. Torres, and J.D. Painter, Nucl. Instr. Meth. B 249 (2006) 789

[11] M. Mayer, R. Fischer, S. Lindig, U. von Toussaint, R.W. Stark, and V. Dose, Nucl. Instr. Meth. B228 (2005) 349

[12] I. Bizyukov and K. Krieger, Review of Scientific Instruments 77 (2006) 043501 [13] M. Mayer, SIMNRA User's Guide, Report IPP 9/113, Max-Planck-Institut für Plasmaphysik, Garching, Germany 1997

[14] R. Huszank, A. Simon, E. Szilágyi, K. Keresztessy, and I. Kovács, Nucl. Instr. Meth. B 267 (2009) 2132

[15] O. Renner and J. Zemek, Czech. J. Phys. B23 (1973) 1273 